\title{
Immune milieu and microbiome of the distal urethra in Ugandan men: impact of penile circumcision and implications for HIV susceptibility
}

Ronald M. Galiwango', Daniel E. Park², Sanja Huibner', Abigail Onos², Maliha Aziz' , Kelsey Roach², Aggrey Anok 3 , James Nnamutete ${ }^{3}$, Yahaya Isabirye ${ }^{3}$, John Bosco Wasswa ${ }^{3}$, Deo Male ${ }^{3}$, Godfrey Kigozi ${ }^{3}$, Aaron A. R. Tobian ${ }^{4}$, Jessica L. Prodger ${ }^{5}$, Cindy M. Liu² and Rupert Kaul ${ }^{1^{*}}$ (1)

\begin{abstract}
Background: Coronal sulcus (CS) anaerobe abundance and IL-8 levels are linked to HIV acquisition, and are dramatically reduced after penile circumcision (PC). The distal urethra may be the site of some HIV acquisition before PC, and presumably most acquisition post PC. We describe the immune milieu and microbiome of the distal urethra in uncircumcised Ugandan men, and define the impact of PC. Participants consisted of HIV-negative, genital symptomfree adult Ugandan men undergoing PC $(n=51)$. Urethral and coronal sulcus swabs were collected at baseline and at 6- and 12-months post-PC. Soluble immune factors were quantified by multiplex ELISA, and bacterial abundance assessed by $16 \mathrm{~S}$ rRNA qPCR and sequencing.
\end{abstract}

Results: At baseline, the urethra was enriched compared to the CS for most cytokines (including IL-8 and MIP-1 $\beta$ ) and soluble E-cadherin (sE-cadherin, an epithelial disruption marker), although CS levels of IL-1a and IL-1 $\beta$ were higher. Baseline total bacterial abundance was $\geq 20$-fold higher in the CS than the urethra (median 27,100 vs. 1200 gene copies/swab, $p=0.001$ ), and anaerobes comprised $58 \%$ of CS bacteria vs. $42 \%$ of urethral bacteria. PC did not alter urethral IL-8 (median 806 at baseline vs. $1130 \mathrm{pg} / \mathrm{ml}$ at 12 months; $p=0.062$ ) and urethral sE-cadherin increased $(113,223 \mathrm{vs} .158,385 \mathrm{pg} / \mathrm{ml}, p=0.009)$, despite five- and sevenfold drops in total bacterial and anaerobe abundance after PC, respectively. However, $\mathrm{PC}$ dramatically reduced CS levels of sE-cadherin $(15,843 \mathrm{vs} .837 \mathrm{pg} / \mathrm{ml}, p<0.001)$ and most cytokines (IL-8; $34 \mathrm{vs} .3 \mathrm{pg} / \mathrm{ml}, p<0.001$ ), while reducing total bacterial and anaerobe abundance by 13 -fold and 60 -fold, respectively (both $P \leq 0.004$ ).

Conclusions: The urethra is immunologically rich with characteristics of an HIV-susceptible tissue site. However, PC had no impact on urethral immunology and may have reduced epithelial integrity, despite modest reductions in total bacteria and anaerobes, suggesting that HIV protection from PC is not mediated via immune or microbiome alterations in the urethra.

Keywords: Microbiome, Cytokines, Coronal sulcus, Penile urethra, Penile circumcision, Uganda, HIV

\footnotetext{
*Correspondence: Rupert.kaul@utoronto.ca

${ }^{1}$ Departments of Immunology and Medicine, University of Toronto, St. George Campus, Medical Sciences Building, 1 King's College Circle, Room 6356, Toronto, Ontario M5S1A8, Canada

Full list of author information is available at the end of the article
}

\section{Background}

Penile circumcision (PC) reduces the risk of human immunodeficiency virus type 1 (HIV) acquisition by almost two-thirds, with protection mediated through 
several proposed physical and biological mechanisms [1-3]. This one-time, cost-effective HIV prevention tool has been rolled out on a large scale in resource-limited regions with a high HIV burden [4]. However, in many regions of sub-Saharan Africa, up to $50 \%$ of eligible men decline the procedure [5-7]. Therefore, a better understanding of the biological mechanism(s) for PC-mediated protection may inform the design of alternative prevention tools focused on uncircumcised men who decline PC.

At least part of the mechanism by which PC protects against HIV infection is stochastic, mediated through the reduced surface area of penile tissues exposed to HIV-containing genital fluids during sex and the direct removal of tissue-associated HIV-susceptible target cells that include activated $\mathrm{CD} 4+\mathrm{T}$ lymphocyte subsets, Langerhans cells, macrophages, and dendritic cells [8, 9]. In addition, foreskin removal obliterates the subpreputial space, where HIV-containing genital fluids may be retained between the glans penis and inner foreskin after sex, thereby prolonging virus-tissue contact. In addition to these physical alterations, surgical obliteration of the subpreputial space via $\mathrm{PC}$ exposes the coronal sulcus (CS) to air, profoundly altering both the penile microbiome composition and immune milieu. The total bacterial load and the proportion of anaerobes is dramatically reduced in the CS after PC, with a shift from anaerobes typically associated with bacterial vaginosis (e.g., Gardnerella, Prevotella, and Peptostreptococcus) toward facultative anaerobic bacteria generally considered to be normal skin flora (e.g., Staphylococcus and Corynebacteria). There are also substantial reductions in the levels of the chemoattractant cytokine interleukin (IL)-8 [9-11], a chemokine which has been directly linked to the increased density of anaerobes in the prepuce, to an increased density of foreskin HIV target cells, and to an increased risk of HIV acquisition risk [9]. This suggests that the microenvironment of the subpreputial space in an uncircumcised man sustains an anaerobic microbiome that induces host tissue inflammation and thereby increases HIV susceptibility [12].

There is still residual HIV acquisition after PC, and the urethra is thought to be the major site for virus acquisition in circumcised men [12]. Indeed, it has been hypothesized that the urethra may be the tissue site for more virus acquisition in uncircumcised men than has been appreciated, and that a major mechanism underpinning PC-mediated protection could be alterations in the microbiome and immunology of the penile urethra, instead of the CS [13]. In keeping with this, the penile urethra contains several potential HIV target cell subsets, including CD4+ CCR5+ macrophages, dendritic cells, and activated CD4+ T cells $[14,15]$ while urethral macrophages may act as an HIV reservoir after HIV acquisition and treatment [16]. Urethral innate and immune defenses include physical flushing during urination, mucus secretion to trap pathogens, antimicrobial factors that include defensins and lysozyme, and immunoglobulin production (especially secretory immunoglobulin A, IgA) [17]. The anatomy of the urethra naturally limits aeration, and it is possible that the overhanging foreskin of an uncircumcised penis might further enhance the anaerobic environment and/or further prolong urethral contact with HIV-containing coital secretions. However, PC does not protect against other urethrally acquired genital infections such as gonorrhea and chlamydia [18], despite reducing the risk of "skin acquired" infections such as human papillomavirus (HPV), herpes simplex virus type 2 (HSV-2), and genital ulcer disease $[19,20]$. In order to assess the impact of PC on the urethral environment and microbiome, we prospectively enrolled Ugandan men presenting for elective PC into a longitudinal clinical protocol, with repeat sampling of the distal urethra and CS prior to and after surgical PC.

\section{Results}

\section{Participant demographics}

A total of 51 participants were enrolled prior to elective PC, with 46 (90\%) and 35 (69\%) of participants attending scheduled 6- and 12-month post-PC follow-up visits. The mean age of participants was 22 years (Table 1); 45 men $(88 \%)$ reported having ever had vaginal intercourse, with $87 \%$ of these reporting multiple $(>1)$ lifetime sexual partners, and just over half reporting multiple female partners within the previous 6 months. A minority of men (4\%) reported antibiotic exposure during the preceding 3 months, and almost all (94\%) reported regular retraction of the foreskin during washing.

\section{Immunology and bacteriology of the urethra and coronal sulcus in uncircumcised men}

At baseline (pre-PC), the immune milieu of the urethra and coronal sulcus was distinct (Table 2). Specifically, the distal urethra was significantly enriched for multiple cytokines and other immune factors compared to the CS, including IL-8, matrix metallopeptidase 9 (MMP-9), macrophage inflammatory protein (MIP)- $1 \beta$, resistin and tissue inhibitor of metalloproteinases (TIMP)-1 (all $P<$ 0.001). Vascular endothelial growth factor (VEGF) levels did not differ between tissue sites $(P>0.10)$, and IL- $1 \alpha$ and IL- $1 \beta$ levels were relatively enriched in the CS compared to the distal urethra $(P<0.001)$ (Table 2$)$. Given the role of epithelial barrier integrity in impairing HIV entry and the potential epithelial disruption induced by inflammatory cytokines [21], we also assayed levels of soluble 
Table 1 Demographics of study participants

\begin{tabular}{|c|c|c|c|}
\hline & & $N$ & Percent \\
\hline Mean age in years (SD) & & $22.18(5.61)$ & \\
\hline \multirow[t]{2}{*}{ Current marital status } & Married/ever married/cohabiting & 10 & 19.6 \\
\hline & Single (never married) & 41 & 80.4 \\
\hline \multirow[t]{2}{*}{ Ever had penile-vaginal sex } & Yes & 45 & 88.2 \\
\hline & No & 6 & 11.8 \\
\hline \multirow[t]{3}{*}{ Current sexual relationship } & Yes & 20 & 39.2 \\
\hline & No & 25 & 49 \\
\hline & Not applicable & 6 & 11.8 \\
\hline \multirow[t]{3}{*}{ Sexual partners, last 6 months } & No partners & 14 & 27.5 \\
\hline & Multiple & 14 & 27.5 \\
\hline & Single & 23 & 45.1 \\
\hline \multirow[t]{3}{*}{ Sexual partners, lifetime } & Single & 6 & 11.8 \\
\hline & Multiple & 39 & 76.5 \\
\hline & Not applicable & 6 & 11.8 \\
\hline \multirow[t]{5}{*}{ Condom use with new partner } & Always & 17 & 37.8 \\
\hline & Most of the time & 9 & 20 \\
\hline & Sometimes & 13 & 28.9 \\
\hline & Rarely & 2 & 4.4 \\
\hline & Never & 4 & 8.9 \\
\hline \multirow[t]{2}{*}{ Antibiotics use within last 3 months } & Yes & 2 & 3.9 \\
\hline & No & 49 & 96.1 \\
\hline \multirow[t]{2}{*}{ Retract foreskin during washing } & Yes & 48 & 94.1 \\
\hline & No & 3 & 5.9 \\
\hline \multirow[t]{5}{*}{ Foreskin washing frequency } & More than once a day & 21 & 41.2 \\
\hline & Daily & 19 & 37.3 \\
\hline & 2-5 times a week & 7 & 13.7 \\
\hline & Once a week & 1 & 2 \\
\hline & Not applicable & 3 & 5.9 \\
\hline
\end{tabular}

Table 2 Pre-circumcision levels of soluble immune factors in the urethra and the coronal sulcus

\begin{tabular}{llll}
\hline Immune factor $(\mathbf{p g} / \mathbf{m l})$ & Urethra (median; Q1-Q3) & Coronal sulcus (median; Q1-Q3) & $\begin{array}{l}\text { P value } \\
\text { (inter-site } \\
\text { difference) }\end{array}$ \\
\hline SE-cadherin & & & $<0.001$ \\
IL-1a & $111,927.83(67,948.42-163,766.44)$ & $15,843.23(4393.86-60,484.44)$ & $<0.001$ \\
IL-1 3 & $45.90(30.48-78.39)$ & $361.12(169.82-529.78)$ & 0.006 \\
IL-8 & $4.45(2.07-11.07)$ & $9.09(3.56-29.60)$ & $<0.001$ \\
MIP-1 3 & $871.51(341.92-1703.33)$ & $33.87(12.75-191.28)$ & $<0.001$ \\
MMP-9 & $13.71(4.25-25.99)$ & $4.19(2.10-6.82)$ & $<0.001$ \\
Resistin & $33,608.41(6138.33-54,037.42)$ & $161.62(52.28-1158.10)$ & $<0.001$ \\
TIMP-1 & $2680.62(691.89-4429.02)$ & $109.30(44.28-911.86)$ & $<0.001$ \\
VEGF & $23,836.69(14,432.54-52,817.63)$ & $2155.23(486.38-5018.20)$ & 0.147 \\
\hline
\end{tabular}

E-cadherin (sE-cadherin), a tight junction protein, and found that these were significantly elevated in the distal urethra compared to CS $(P<0.001)$ (Table 2$)$. In the CS, baseline levels of $\mathrm{sE}$-cadherin correlated strongly with several proinflammatory biomarkers, including increased IL-8 levels $(r=0.624 ; P<0.0001)$, while no correlation was observed between levels of $\mathrm{sE-cadherin} \mathrm{and} \mathrm{inflam-}$ matory biomarkers in the distal urethra. 
In contrast to most immune factors, baseline (pre-PC) total bacterial abundance, defined as the $16 \mathrm{~S}$ ribosomal RNA copy number/swab, was 17 -fold higher in the CS than the distal urethra (median $25,980,000$ vs. $1,468,000$ gene copies/swab, $p=0.001$ ). In addition, anaerobes constituted a higher proportion of the CS microbiome, where they comprised $58 \%$ of the microbiome, compared to $42 \%$ in the distal urethra (Fig. 1A, Table 3). The composition of the urethral microbiome was also quite distinct from the CS microbiome, with Streptococcus being
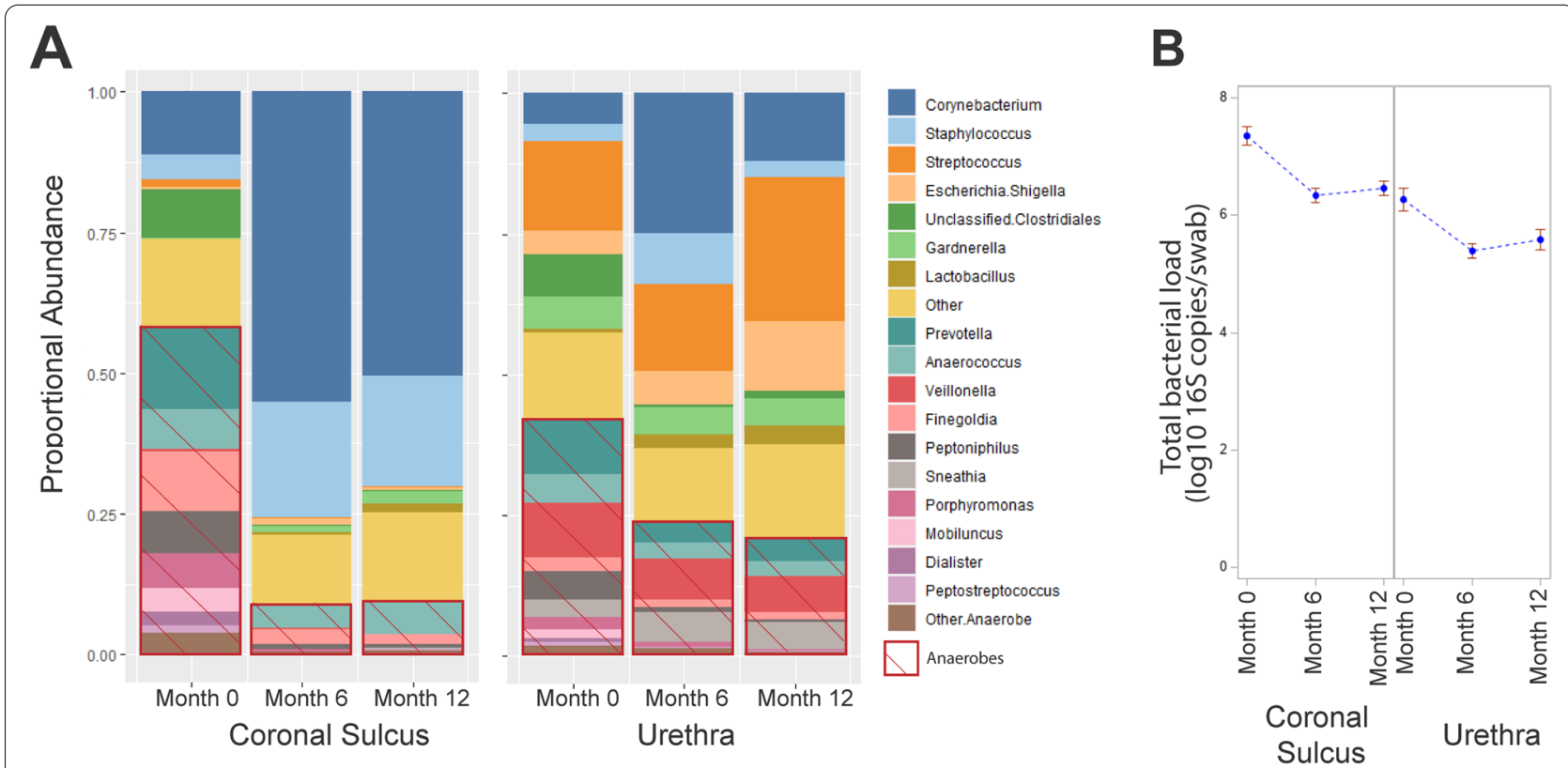

Fig. 1 Impact of PC on microbiome composition and total bacterial abundance at the urethra and the coronal sulcus. A Stacked barcharts display proportional abundance of bacteria at the coronal sulcus and urethra, grouped by oxygen dependence into aerobes and anaerobes (hatched red box) at baseline, 6 months, and 12 months ( $N=51,45$, and 35 participants respectively). B Mean total absolute bacterial abundance (log 10 gene copies per swab) and standard errors at the coronal sulcus and urethra, by study time point

Table 3 Baseline bacterial proportional and absolute abundance of prevalent genera in the urethra and the coronal sulcus

\begin{tabular}{|c|c|c|c|c|c|}
\hline \multirow[t]{3}{*}{ Oxygen tolerance ${ }^{* *}$} & \multirow[t]{3}{*}{ Taxa } & \multicolumn{2}{|c|}{ Mean proportional abundance } & \multicolumn{2}{|c|}{$\begin{array}{l}\text { Median } \log _{10} \text { absolute abundance* } \\
\text { (Q1-Q3) }\end{array}$} \\
\hline & & Coronal sulcus & Urethra & Coronal sulcus & Urethra \\
\hline & & $n=47$ & $n=46$ & $n=47$ & $n=46$ \\
\hline AN & Prevotella & $14.6 \%$ & $9.5 \%$ & $6.3(4.9-7.1)$ & $4.6(3.0-5.8)$ \\
\hline AN & Finegoldia & $10.6 \%$ & $2.4 \%$ & $5.9(5.1-6.8)$ & $4.2(3.2-5.3)$ \\
\hline AN & Peptoniphilus & $7.7 \%$ & $5.0 \%$ & $6.1(5.1-6.9)$ & $4.6(3.5-5.5)$ \\
\hline AN & Anaerococcus & $7.2 \%$ & $5.2 \%$ & $6.0(5.1-6.9)$ & $4.6(3.7-5.7)$ \\
\hline AN & Dialister & $2.6 \%$ & $0.7 \%$ & $5.1(0.0-6.0)$ & $3.2(0.0-4.4)$ \\
\hline AN & Peptostreptococcus & $1.3 \%$ & $0.8 \%$ & $0(0.0-4.3)$ & $0(0.0-3.6)$ \\
\hline AN & Veillonella & $0.3 \%$ & $9.7 \%$ & $0(0.0-4.4)$ & $0(0.0-5.7)$ \\
\hline FAN & Corynebacterium & $11.2 \%$ & $5.5 \%$ & $5.4(4.2-6.5)$ & $4.4(2.9-5.3)$ \\
\hline AN & Unclassified Clostridiales & $8.7 \%$ & $7.5 \%$ & $5.9(3.7-6.9)$ & $3.6(0.0-5.3)$ \\
\hline FAN & Staphylococcus & $4.3 \%$ & $2.9 \%$ & $4.3(0.0-5.7)$ & $2.8(0.0-4.4)$ \\
\hline FAN & Streptococcus & $1.4 \%$ & $16.1 \%$ & $0(0.0-4.7)$ & $5.2(3.9-6.3)$ \\
\hline FAN & Gardnerella & $0.1 \%$ & $5.8 \%$ & $0(0.0-0.0)$ & $0.0(0.0-5.0)$ \\
\hline FAN/AN/MAE & Lactobacillus & $0.0 \%$ & $0.7 \%$ & $0(0.0-0.0)$ & $0.0(0.0-0.0)$ \\
\hline
\end{tabular}

*16S rRNA gene copies per swab

${ }^{*} A N$ strictly anaerobic, $A E$ strictly aerobic, FAN facultative anaerobic, MAE microaerophilic 
the most abundant and comprising an average of $16 \%$ of urethral bacteria, followed by Veillonella, Prevotella, unclassified Clostridiales, Corynebacterium, and Anaerococcus at $10 \%, 10 \%, 8 \%, 6 \%$, and $5 \%$ respectively. By contrast, Prevotella was the most abundant CS bacteria (mean 15\%), followed by Corynebacterium, Finegoldia, unclassified Clostridiales, and Peptoniphilus at $11 \%, 11 \%$, $9 \%$, and $8 \%$ respectively (Fig. $1 \mathrm{~A}$, Table 3). Notably, key female genital tract bacteria, particularly Lactobacillus and Gardnerella, were rare at both penile sites.

\section{Differential impact of penile circumcision on the coronal sulcus versus distal urethra immune milieu}

Levels of the proinflammatory chemoattractant IL-8 at the CS fell significantly 6 months after PC (median 5.39 vs. $33.87 \mathrm{pg} / \mathrm{ml}, p<0.001$ ), with further reductions at 12 months ( 3.41 vs. $33.87 \mathrm{pg} / \mathrm{ml}, p<0.001$; Fig. 2 A). Similar falls after PC were observed for IL-1 $\beta$, resistin, sEcadherin, and VEGF (Fig. 2B-C, E-F), although there was no impact of PC on CS levels of MIP-1 $\beta$ or MMP-9 (data not shown). Unexpectedly, levels of the proinflammatory cytokine IL-1 $\alpha$ in the coronal sulcus actually increased significantly at both 6 months $(788.48$ vs. $358.77 \mathrm{pg} / \mathrm{ml}$; $p<0.001$ ) and 12 months (538.02 vs. $358.77 \mathrm{pg} / \mathrm{ml}, p=$ 0.018; Fig. 2D) after PC.

In contrast to the profound immune alterations seen at the coronal sulcus, there was unexpectedly little impact of PC on the urethral immune milieu. Urethral IL-8 levels were unaltered at 6 months post PC (754.96 vs. 805.59 $\mathrm{pg} / \mathrm{ml} ; P=0.906)$ and actually tended to increase at 12 months (1130.21 vs. $805.59 \mathrm{pg} / \mathrm{ml} ; P=0.062$; Fig. 3A). Other immune biomarkers remained unchanged, with the exception of a transient increase in urethral IL- $1 \alpha$ levels at 6 months (67.81 vs. $45.90 \mathrm{pg} / \mathrm{ml} ; P=0.032$; Fig. 3D) and a sustained increase in levels of sE-cadherin at both 6 months $(179,590.44$ vs. $113,223.06 \mathrm{pg} / \mathrm{ml} ; P=0.032)$ and 12 months $(158,384.77$ vs. $113,223.06 \mathrm{pg} / \mathrm{ml} ; P=0.009$; Fig. $3 \mathrm{E})$.

\section{Penile circumcision and the coronal sulcus and urethral microbiome}

Total bacterial load remained higher at the CS than the urethra at follow-up visits (Fig. 1B), although there were significant reductions at both sites post-PC, with a disproportionate reduction in anaerobic taxa (Fig. 1A). At the CS, there was a $>13$-fold reduction in total bacterial load at both 6 months and 12 months, while reductions in the urethra were less marked (sixfold) and only significant at 6 months (Fig. 1B).

Broadly, post-PC, there was a shift toward "skin-associated" gram-positive aerobes at the CS (from 58\% anaerobe dominance at baseline to $90 \%$ aerobe dominance at 12 months), largely composed of Corynebacterium (50\%) and Staphylococcus (20\%). At the distal urethra, the aerobe Streptococcus dominated the microbiome both pre- and post-PC (16\% and $26 \%$ respectively). PC increased the proportion of Corynebacterium both at the $\mathrm{CS}$ and in the urethra (Fig. 1A).

Circumcision substantially reduced the absolute abundance of anaerobic taxa at both penile tissue sites. This was most profound in the CS, where there was a 60 -fold drop (from 12,733,000 to 204,098 gene copies per swab; $P<0.001$ ) but was also significant in the urethra (14-fold drop). Specifically, at the CS, PC significantly reduced the absolute abundances of several specific bacterial genera linked to BV and HIV risk in both men and women. PC profoundly reduced the abundance of most BV-associated bacterial genera in the CS at 12 months, particularly Prevotella (median 0 vs. 2,159,328 gene copies per swab, $P<0.0001$; Fig. 4A). While there had been a marked proportionate increase in gram positive aerobes after PC, their absolute abundance did not significantly change (e.g., Corynebacterium, 894,487 vs. $272,834, P=0.512$; and Staphylococcus, 370,878 vs. $21,426, P=0.120$; Fig. $4 \mathrm{E}$, $G)$. However, there were similar sustained drops in the absolute abundance of several BV-associated taxa, including Prevotella and Peptoniphilus (0 vs. 40,604 gene copies/swab, $P=0.02$; and 0 vs. 34,433 gene copies/swab, $P<$ 0.0001 respectively; Fig. 4B, D). Similar to the CS, despite the proportionate change, there was no increase in the absolute abundance of skin commensals Staphylococcus and Corynebacterium at the urethra $(P>0.5 ;$ Fig. $4 \mathrm{~F}, \mathrm{H})$.

\section{Immune associations of specific penile bacterial genera}

The bacterial vaginosis (BV)-associated taxa Prevotella and Dialister in the CS have been most strongly associated with HIV seroconversion in uncircumcised men, while a CS microbiome enriched for skin-associated taxa such as Corynebacterium and Staphylococcus is associated with HIV protection after PC [10]. Therefore, we next assessed how the absolute abundances of these taxa correlated with immune parameters at both the CS and urethra prior to PC, specifically with IL-8 (a chemoattractant cytokine linked to penile HIV acquisition) and E-cadherin (a tight junction protein linked to epithelial disruption).

At the CS, Dialister abundance correlated positively with levels of IL-8 and sE-cadherin (Spearman coefficients $0.400, p=0.006$ and $0.659, p<0.0001$; Fig. 5), while Corynebacterium abundance was negatively correlated with both parameters (Spearman coefficient -0.326 , $p=0.029$ and $-0.307, p=0.037$; Fig. 5). Likewise, CS Prevotella abundance tended to be positively associated with these immune parameters (Spearman coefficients 0.183, $p=0.229$ and 0.600, $p<0.0001$ ) and Staphylococcus abundance negatively (Spearman coefficients -0.214 , $p=0.158$ and $-0.420, p=0.004)$. Bacterial-immune associations were weaker in the distal urethra, although 


\section{A}

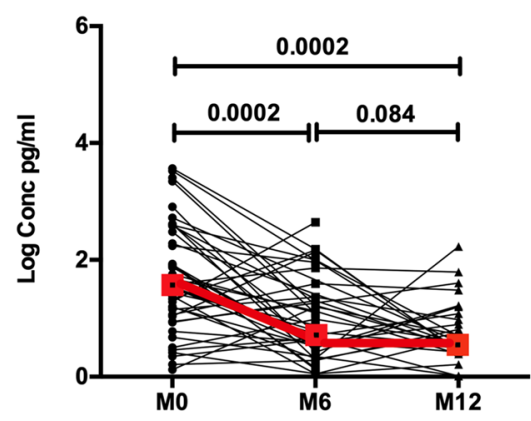

C

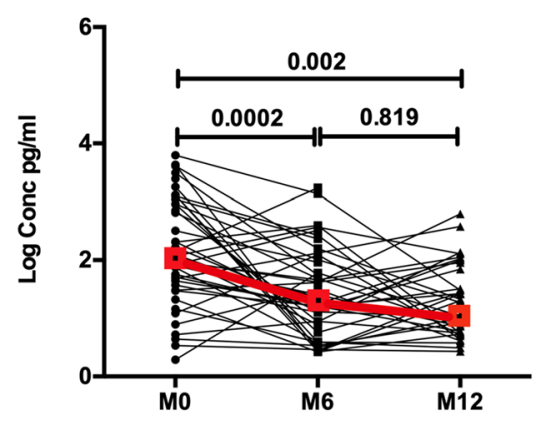

E

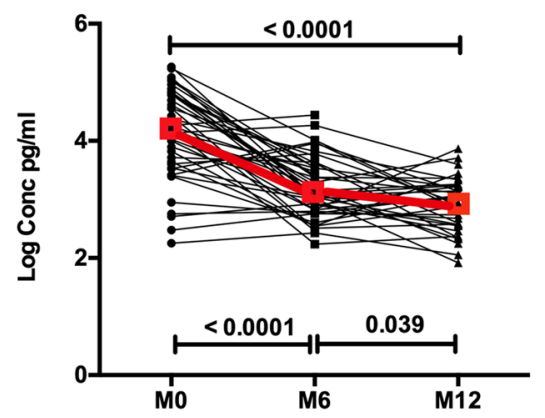

B

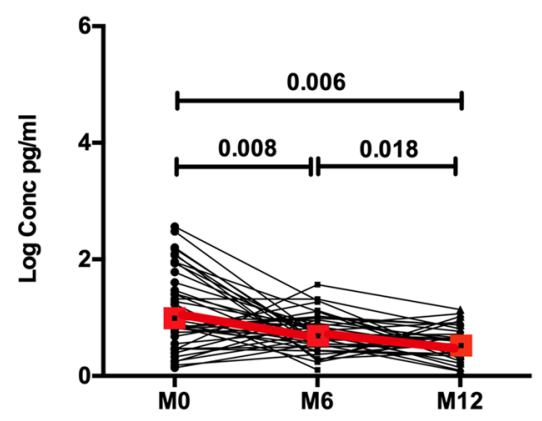

D

IL-1 $\alpha$

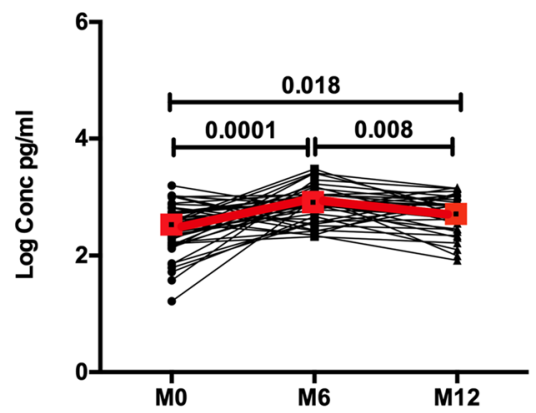

$\mathbf{F}$

VEGF

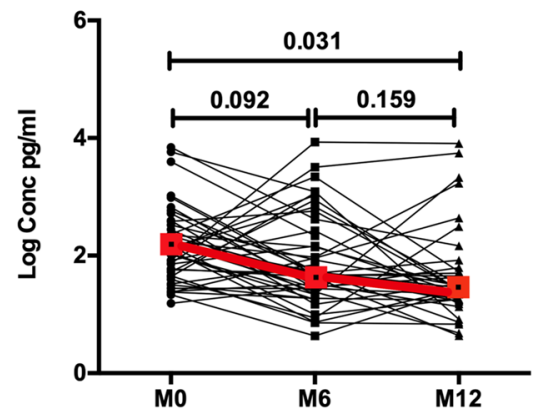

Fig. 2 Impact of penile circumcision on immune parameters at the coronal sulcus. Plots show log transformed concentrations (in pg/ml) with inter-quartile ranges of IL-8 (A), IL-1 $\beta(\mathbf{B})$, resistin $(\mathbf{C})$, IL-1a (D), sE-cadherin $(\mathbf{E})$, and VEGF $(\mathbf{F})$ at pre-circumcision $(N=51), 6 \mathrm{month}(\mathrm{N}=45)$, and 12 month $(N=35)$ post circumcision. Red boxes denote the median concentration at each visit time point

Prevotella abundance was again positively associated with IL-8 levels (Spearman coefficients $0.350, p=0.018$ ), and both Corynebacterium and Staphylococcus abundance with reduced sE-cadherin (Spearman coefficient -0.304 , $p=0.043$, Fig. 5 and $-0.306, p=0.041$ respectively).

Overall, at the CS of uncircumcised men, skinassociated bacterial taxa were associated with less inflammation and enhanced epithelial integrity, while seroconversion-associated taxa were associated with more inflammation and reduced integrity; similar but weaker associations were seen in the distal urethra.

\section{Discussion}

The HIV protection that is afforded by PC [1-3] may be mediated via several mechanisms, including direct removal of HIV-susceptible foreskin tissue [22, 23] and 

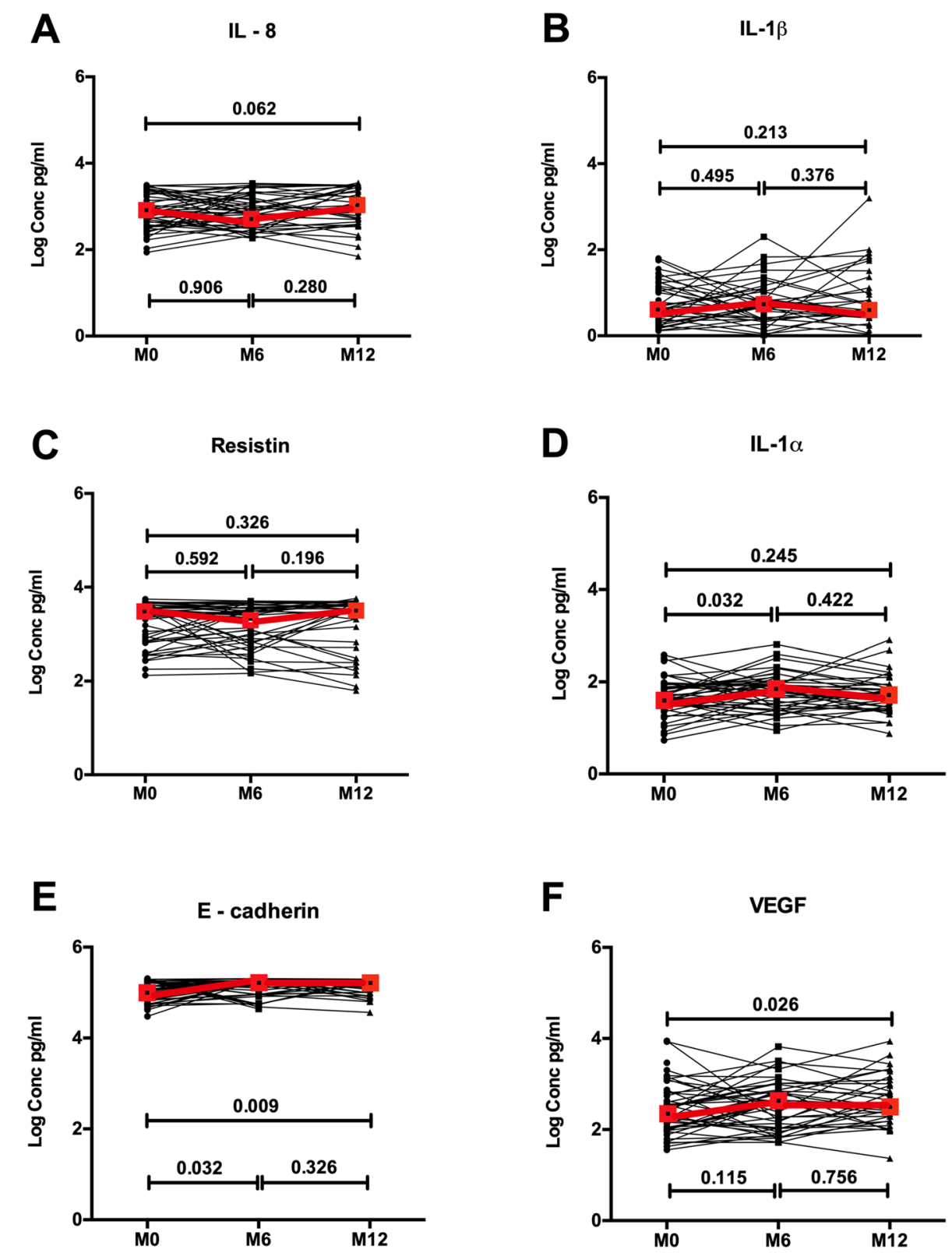

Fig. 3 Impact of penile circumcision on immune parameters in the distal urethra. Plots show log transformed concentrations (in pg/ml) with inter-quartile ranges of IL-8 (A), IL-1 $\beta(\mathbf{B})$, resistin $(\mathbf{C})$, IL-1a (D), E-cadherin $(\mathbf{E})$, and VEGF $(\mathbf{F})$ pre-circumcision $(N=51), 6$ months $(N=45)$, and 12 months $(N=35)$ post circumcision. Red boxes denote the median concentration at each time point

reductions in inflammatory cytokines and pro-inflammatory anaerobes in the coronal sulcus [9]. The urethra is assumed to be the site of residual penile HIV acquisition after PC, but it has been hypothesized that this tissue site may play a greater role than appreciated prior to $\mathrm{PC}$, due to urethral immune and microbiome parameters induced by an overhanging foreskin, so that HIV protection post-PC might relate to an altered urethral immune or microbiological milieu [13]. We addressed this research question in a cohort of adult Ugandan men presenting for elective PC. As expected from prior studies [9, 10, 24], we demonstrated that PC dramatically reduced most proinflammatory anaerobes and inflammatory cytokines/ chemokines in the coronal sulcus, with simultaneous reductions in soluble E-cadherin suggesting enhanced epithelial integrity. Prior to PC, the microbiome and immune milieu of the distal urethra were quite distinct to the coronal sulcus, and the impact of PC also differed at this tissue 


\section{Coronal sulcus}
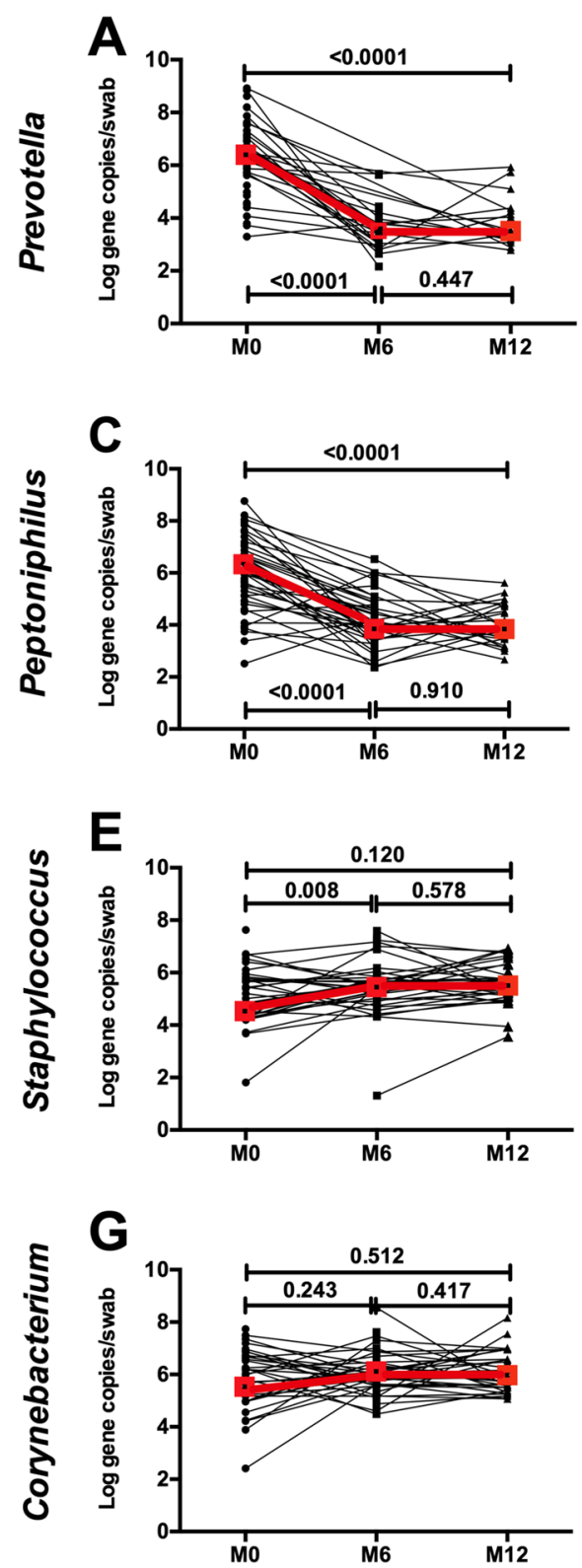

\section{Urethra}
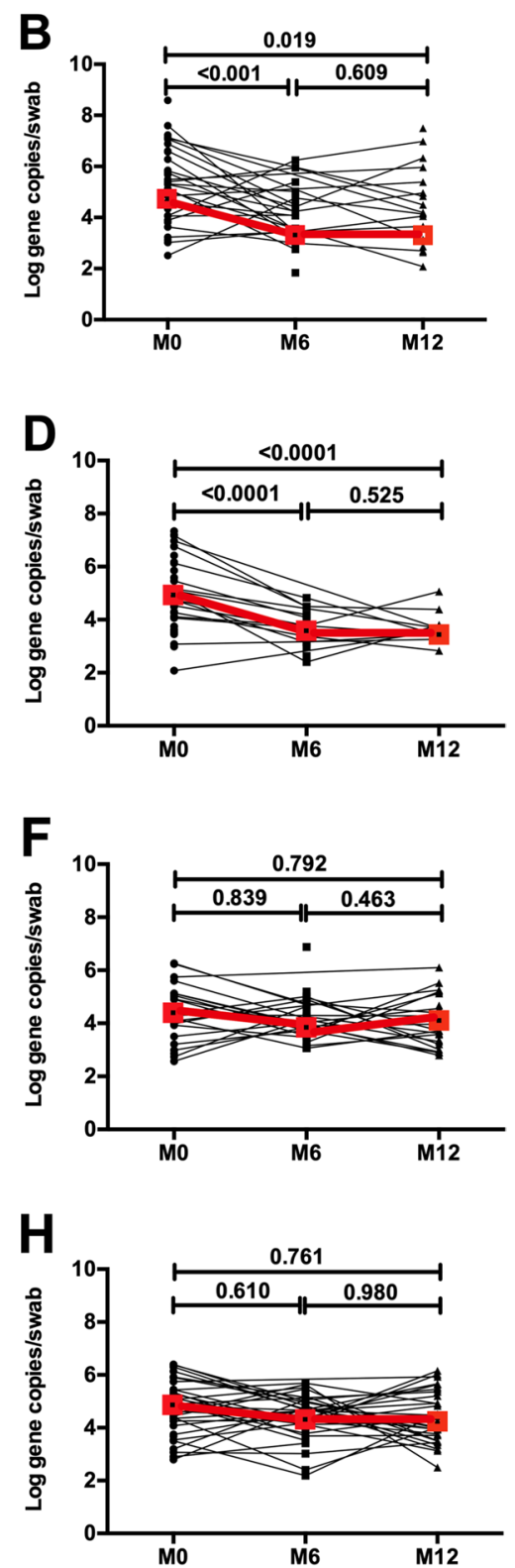

Fig. 4 Impact of PC on bacterial absolute abundance at the urethra and the coronal sulcus. Absolute bacterial abundances in the CS and urethra of the selected genera Prevotella (A, B), Peptoniphilus $(\mathbf{C}, \mathbf{D})$, Staphylococcus $(\mathbf{E}, \mathbf{F})$, Corynebacterium $(\mathbf{G}, \mathbf{H})$ were compared across study visits $(N=51$, 45, and 35 participants respectively). Red boxes denote the median concentration at each time point

site. The urethra of the uncircumcised penis had a much lower bacterial load than the CS, with a smaller proportion of anaerobes, and there were higher levels of IL-8, sE-cadherin, and most other soluble immune parameters (with the exception of IL1 $\alpha$ and IL1 $\beta$ ). However, while PC substantially reduced the bacterial load and proportionately enriched the microbiome of both tissue sites for skin-associated gram-positive aerobes, this was much less marked in the urethra than the coronal sulcus, and PC had a minimal impact on urethral cytokines and chemokines while increasing urethral sE-cadherin levels. Overall, these results suggest that HIV protection following PC is not likely to be mediated through alterations in the urethral microbiome or urethral immune milieu.

Differences at the urethra and coronal sulcus in levels of proinflammatory/chemoattractant biomarkers such 


\section{Coronal sulcus}

A
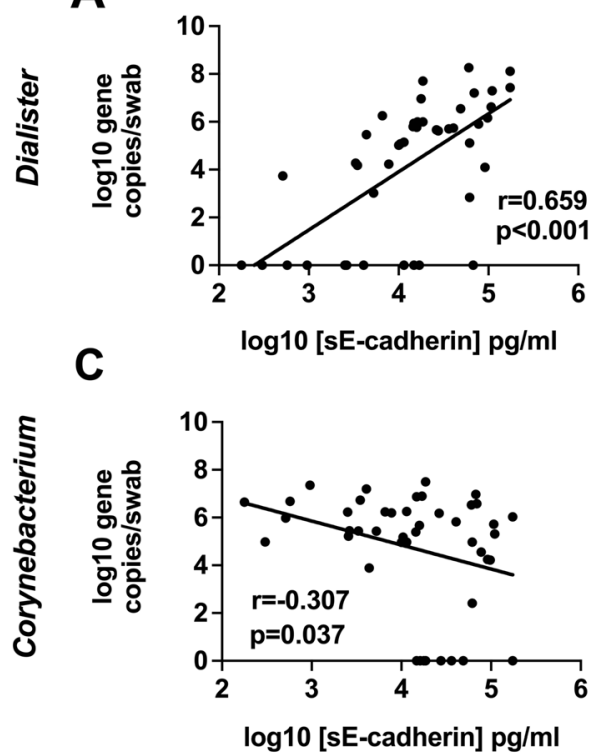

E

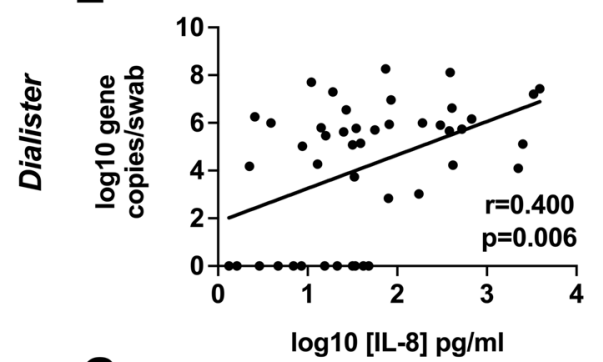

G

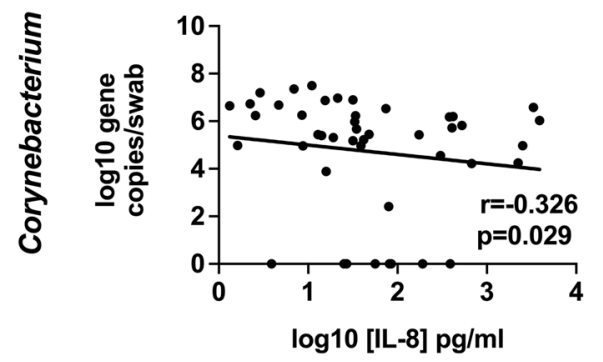

B

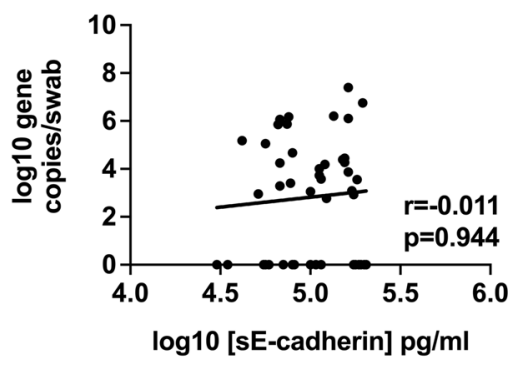

D

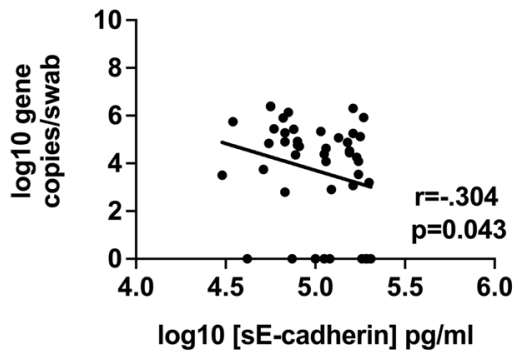

F

H
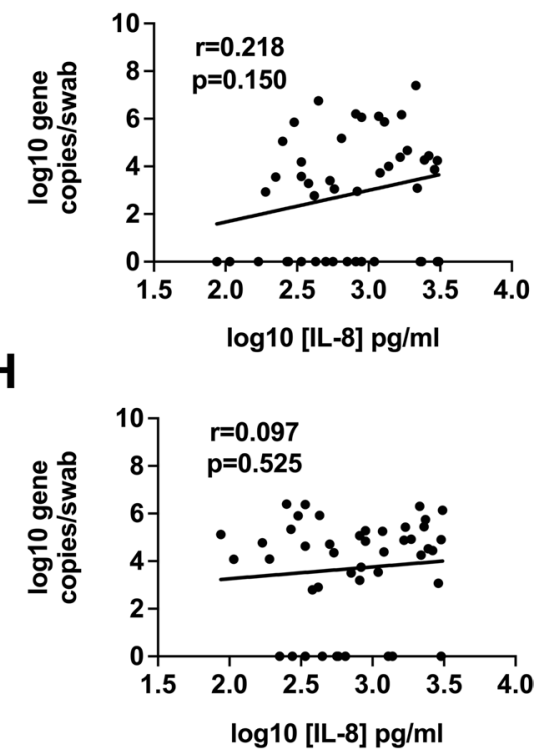

Fig. 5 Associations between penile bacteria abundance and immune parameters at the urethra and coronal sulcus of uncircumcised men. Absolute abundances of selected penile bacteria Dialister and Corynebacterium showed distinct correlations with sE-cadherin and IL-8 at the urethra versus the coronal sulcus prior to PC. At the coronal sulcus, Dialister tended to be positively $(\mathbf{A}, \mathbf{C})$ and Corynebacterium negatively $(\mathbf{E}, \mathbf{G})$ associated; at the distal urethra similar associations were seen, but often weaker or non-significant $(\mathbf{B}, \mathbf{D}, \mathbf{F}, \mathbf{H} ; N=51)$

as IL-8, and the contrasting effects of $\mathrm{PC}$ on the local immune milieu, may have important implications for HIV susceptibility. IL-8 is produced by epithelial and antigen presenting cells, and serves as both a chemoattractant and proinflammatory cytokine [25, 26]. Elevated genital IL-8 levels are associated with enhanced HIV acquisition in both women $[27,28]$ and uncircumcised men [9], where they correlate with an increased density of CD4+ T cell targets in the endocervix and foreskin, respectively $[9,29]$. Although baseline urethral IL-8 levels were over 20 -fold higher in the urethra than the coronal sulcus, urethral levels of IL-8 and other cytokines/ 
chemokines were unaltered by $\mathrm{PC}$, despite modest reductions in the urethral bacterial load and a microbiome shift toward skin-associated gram-positive aerobes. In addition, no association was seen between urethral anaerobes and local inflammation, even in those men with the highest anaerobic bacterial load at baseline. It remains unclear whether urethral anaerobes simply do not induce inflammation at this site, whether additional factors such as increased local epithelial disruption (evidenced by high E-cadherin levels) have a greater contribution to inflammation, or whether cytokines induced by inflammation are not able to accumulate due to intermittent "flushing" during urination.

Soluble E-cadherin levels appear to be a biomarker of epithelial disruption at other mucosal sites [30-32]. This transmembrane glycoprotein connects epithelial cells at adherens junctions and is integral in mediating cell adhesion and contact inhibition of proliferation [33]; therefore, elevated levels indicate disrupted cell-cell junctions and a lack of epithelial integrity. Prior to PC, urethral sE-cadherin levels were almost tenfold higher than in the coronal sulcus, despite a lower total bacterial load and reduced anaerobic burden. While G. vaginalis induced higher soluble sE-cadherin levels and the expression of IL-8 and IL-6 in both the murine genital tract [32] and a human ex vivo cervicovaginal model [30], we only observed a correlation between inflammatory anaerobes, IL-8 levels and sE-cadherin in the coronal sulcus, but not the urethra. In addition, there was an unexpected increase in urethral sE-cadherin levels post- $\mathrm{PC}$, which we hypothesize, may relate to minor post-surgical irritation of the now "unshielded" distal urethra (e.g., through rubbing on clothing).

Finally, we observed an unexpected and substantial increase IL- $1 \alpha$ levels after PC at the CS, despite profound reductions in pro-inflammatory anaerobes and a reduction in other cytokines and chemokines (including IL-8). Although perhaps not intuitive, this may make biological sense: while IL-1 $\alpha$ is predominantly thought of as a proinflammatory cytokine, it also mediates several epidermal barrier functions that include stimulation of keratinocyte proliferation and differentiation, upregulation of genes associated with cell adhesion and the synthesis of lipids needed for the formation and maturation of the stratum corneum [34-36]. As a result, all epithelial cells (especially epidermal cells) constitutively express relatively high levels of IL- $1 \alpha$, and it is possible that elevated levels may relate to healing of the epidermal barrier [37, 38].

There are some limitations to our study that merit discussion. We were not able to directly link immune/ microbiome changes seen at either penile tissue site with actual HIV acquisition, since this would have required a much larger sample size. However, profound immune and bacterial changes were seen at both sites, and the lack of alteration in urethral immunology postPC, coupled with elevated sE-cadherin, strongly suggests that the protection mediated by $\mathrm{PC}$ is unlikely to be mediated urethrally. Nonetheless, additional studies will be needed to confirm and expand our observations, potentially with the examination of additional immune markers and an assessment of PC impact on other microbes such as fungi and viruses. Furthermore, $\mathrm{sE}$-cadherin is an indirect marker of epithelial disruption, and ideally future studies would correlate this with direct microscopic evidence of epithelial damage. We were unable to assess immune cell populations in the urethra, such as $\mathrm{T}$ cells, dendritic cell subsets, neutrophils, and other innate immune cells, due to practical difficulties in tissue sampling at this site. However, previous studies using cadaveric or surgical specimens have demonstrated that many of these cell subsets are abundant in the urethra $[15,39]$, can serve as a reservoir for HIV in infected men taking ART [16], and would be expected to be profoundly altered by the local microbiome and immune milieu.

\section{Conclusions}

In summary, PC does not alter urethral immunology and may reduce urethral epithelial integrity, despite reductions in urethral bacterial load and a modest reduction in the relative proportion of anaerobes. In contrast, PC dramatically reduced the CS bacterial load and anaerobe proportion, enhanced epithelial integrity in the CS, and reduced most CS inflammatory chemokines/cytokines. This suggests that HIV protection post-PC is mediated by the removal of inflamed, HIV-susceptible foreskin tissues rather than by immunologic or microbiome alterations in the urethra.

\section{Methods}

Study enrollments follow-up visits and sample processing Study participants consisted of uncircumcised HIVnegative Ugandan men who did not have any genital STI symptoms, aged at least 18 years electively presenting at the Rakai Health Sciences Program for voluntary male medical PC to reduce their HIV risk. Penile samples were collected at baseline (pre-PC) and at 6- and 12-months following PC. A social-behavioral questionnaire was also administered at each study visit. The study clinician used sterile polyester tips (Puritan Medical Products, ME, USA) premoistened in phosphate-buffered saline (PBS) to swab the inner foreskin (pre-PC) or coronal sulcus (post-PC), and nylon flocked urethral swabs (Hardy Diagnostics, CA, USA) were used 
to swab the distal urethra. Swabs were immediately placed into $500 \mu \mathrm{L}$ of PBS and transferred to the laboratory on ice. In the laboratory, swabs were vigorously vortexed for $60 \mathrm{~s}$ and then the swab head was inverted prior to a quick spin to dry out the swab, which was then discarded. Each sample tube was pulse-vortexed and two aliquots each containing $250 \mu \mathrm{L}$ of supernatant were logged and frozen at $-80{ }^{\circ} \mathrm{C}$.

\section{Multiplex chemiluminescent ELISA}

Levels of 9 soluble immune biomarkers were assayed using a multiplex electro-chemiluminescence ELISA platform (Meso Scale Discovery, Rockville, MD) by research personnel fully blinded to PC status. The biomarker panel included the prototypic proinflammatory cytokines interleukin 1 alpha (IL-1 $\alpha$ ), and interleukin 1 beta (IL-1ß); the chemoattractant chemokines interleukin 8 (IL-8) and macrophage inflammatory protein 1 beta (MIP-1 $\beta)$; and a biomarker of epithelial integrity/breakdown (E-cadherin). Other exploratory analytes on the panel included resistin, an atypical proinflammatory biomarker, and the novel biomarkers tissue inhibitor of metalloproteases 1 (TIMP-1), vascular endothelial growth factor (VEGF), and matrix metalloproteinase 9 (MMP-9). Analyte concentrations within each sample were calculated from a standard curve generated using serial dilutions of stock analyte from the manufacturer and this was prepared for each plate run. Lower limits of detection derived from each plate run were applied to samples flagged as below detection for both duplicates irrespective of the coefficient of variation. All samples belonging to a given individual were run on the same plate to limit any potential impact of plate-plate variation. On each plate, a frozen control media aliquot was plated to monitor inter plate/run variability. Our primary immune endpoint was IL-8, since coronal sulcus levels of IL-8 were the strongest immune predictor of HIV acquisition in uncircumcised Ugandan men.

\section{Microbiome laboratory analysis and controls used}

DNA was extracted from $80 \mu \mathrm{L}$ of diluted swab eluent using a combination of enzymatic and chemical lysis. Briefly, each sample was treated with an enzymatic cocktail containing $122 \mu \mathrm{L}$ Tris-EDTA, $50 \mu \mathrm{L} 10 \mathrm{mg} / \mathrm{mL}$ lysozyme (L6876-1G, Sigma-Aldrich), $4 \mu \mathrm{L} 25 \mathrm{KU} / \mathrm{mL}$ mutanolysin (M4782-5KU, Sigma-Aldrich), and $3 \mu \mathrm{L} 4$ $\mathrm{U} / \mu \mathrm{L}$ lysostaphin (SAE0091-2MG, Sigma-Aldrich) at 37 ${ }^{\circ} \mathrm{C}$ for $1 \mathrm{~h}$, followed by extraction using MagMax DNA Multi-Sample Ultra 2.0 Kit (including Proteinase K treatment) with $80 \mu \mathrm{L}$ final elution volume. Penile microbiome analysis was characterized by $16 \mathrm{~S}$ rRNA gene-based broad-range real-time PCR [40] and sequencing. The sequencing analysis was performed using a modified protocol from Fadrosh et al. [41] with forward (341F) and reverse (786R) primers from Liu et al. [40]. Sequencing was performed on MiSeq platform using MiSeq Reagent Kit v3 (600 cycle).

During processing, primer sequences were removed using cutadapt v2.4 [42] and the resultant sequences were quality trimmed using Trimmomatic v0.39 [43]. DADA2 v1.10 [44] modules were used for reads-filtering, chimera check, and inferred error models to identify amplicon sequence variants (ASVs). The ASVs were classified at each taxonomic level at $80 \%$ bootstrap confidence level using the Naïve Bayesian Classifier (v.2.12) [45]. Classification results for each sample were enumerated to generate an abundance matrix for analysis. Additional details can be found at https://github.com/araclab/mb_analysis.

Using the resultant $\mathrm{qPCR}$ and sequencing outputs, absolute abundance of each penile bacterial genus and species was calculated as: Absolute abundance of a taxon per swab $=$ Total bacterial load per swab (measured by qPCR as total copies of $16 \mathrm{~S}$ rRNA gene per swab) $\times$ proportional abundance of the given taxon (measured by sequencing as the number of sequences assigned to a taxon in a given sample, divided by the total number of sequences obtained for the sample). Sequence data for this study can be accessed at SRA project number PRJNA738496.

Negative extraction control (NEC) is included with each batch of extraction and analyzed by qPCR, where $\mathrm{Cp}>$ 33-35 are considered acceptable. NECs are further included in sequencing analysis. No template control (NTC) and positive template controls (PTC) are included for each amplicon PCR plate and analyzed to assess cross-contamination during PCR and verify PCR performance.

\section{Microbiome statistical analysis}

Intra-individual paired analysis was performed using the Wilcoxon-matched pairs signed-rank test in SPSS version 24 (Armonk, New York, USA). Intra-individual baseline cytokine comparisons were performed between the two sites (coronal sulcus vs. urethra) as well as cytokine levels at baseline compared to months 6 and 12. Non-parametric Spearman rank-order correlation was run between specific cytokines/parameters with probable biological interactions. Analyses were visualized using GraphPad Prism version 6 (La Jolla, CA, USA). Missing data (e.g., arising from a missed visit) was excluded from all paired intra-individual analysis. 


\section{Supplementary Information}

The online version contains supplementary material available at https://doi org/10.1186/s40168-021-01185-9.

\section{Acknowledgements}

We thank all the men who kindly participated in the study and staff at the Rakai Health Sciences Program for their help in study implementation.

\section{Authors' contributions}

RMG and RK conceived the study, planned experiments, and drafted the manuscript. SH performed the multiplex cytokine assays. DP, AO, MA, KR, and $\mathrm{CL}$ performed the microbiome assays, and contributed to data analysis. AA, JN, YI, JBW, DM, and GK implemented participant enrollments, follow-up, and data collection. AART, JLP, and CL provided critical feedback, contributed to data analysis and manuscript drafting. All authors critically reviewed the draft of the paper and approved the final version of the manuscript.

\section{Funding}

This work was supported by the Canadian Institutes for Health Research (TMI-138656 to RK), the Fogarty HIV Research Training Program of the National Institutes of Health (4D43TW009578-04 to RMG), and RK is supported by the University of Toronto/OHTN Endowed Chair in HIV Research. This work was also supported in part by extramural funding from the National Institutes of Health (1R01Al128779 to AART) and R01A123002-01A1 (to CL). The funders had no role in the study design, data collection and analysis, decision to publish, or preparation of the manuscript.

\section{Availability of data and materials}

Sequence data for this study can be accessed at SRA project number PRJNA738496. Details for the bioinformatics analyses can be found at https:// github.com/araclab/mb_analysis. Please contact author for additional requests.

\section{Declarations}

\section{Ethics approval and consent to participate}

The Research and Ethics Board (REB) of the Uganda Virus Research Institute in Entebbe, Uganda, and the Institutional Review Board at the University of Toronto in Canada provided review, ethical oversight, and approval of the study proposal. All study participants gave written informed consent in accordance with the Declaration of Helsinki.

\section{Consent for publication}

Not applicable

\section{Competing interests}

The authors declare that they have no competing interests.

\section{Author details \\ ${ }^{1}$ Departments of Immunology and Medicine, University of Toronto, St. George Campus, Medical Sciences Building, 1 King's College Circle, Room 6356, Toronto, Ontario M5S1A8, Canada. ${ }^{2}$ George Washington Milken Institute School of Public Health, Washington, DC, USA. ${ }^{3}$ Rakai Health Sciences Program, Kalisizo, Uganda. ${ }^{4}$ Johns Hopkins University, Baltimore, MD, USA. ${ }^{5}$ Western University, London, Ontario, Canada.}

Received: 5 December 2020 Accepted: 1 November 2021 Published online: 18 January 2022

\section{References}

1. Auvert B, Taljaard D, Lagarde E, Sobngwi-Tambekou J, Sitta R, Puren A. Randomized, controlled intervention trial of male circumcision for reduction of HIV infection risk: the ANRS 1265 Trial. PLoS Med. 2005;2(11):e298.

2. Bailey RC, Moses S, Parker CB, Agot K, Maclean I, Krieger JN, et al. Male circumcision for HIV prevention in young men in Kisumu, Kenya: a randomised controlled trial. Lancet. 2007;369(9562):643-56.
3. Gray RH, Kigozi G, Serwadda D, Makumbi F, Watya S, Nalugoda F, et al Male circumcision for HIV prevention in men in Rakai, Uganda: a randomised trial. Lancet. 2007:369(9562):657-66.

4. Njeuhmeli E, Forsythe S, Reed J, Opuni M, Bollinger L, Heard N, et al. Voluntary medical male circumcision: modeling the impact and cost of expanding male circumcision for HIV prevention in eastern and southern Africa. PLoS Medicine. 2011;8(11):e1001132.

5. Ledikwe JH, Nyanga RO, Hagon J, Grignon JS, Mpofu M, Semo BW. Scaling-up voluntary medical male circumcision - what have we learned? HIV AIDS (Auckl). 2014;6:139-46.

6. Moyo S, Mhloyi M, Chevo T, Rusinga O: Men's attitudes: a hindrance to the demand for voluntary medical male circumcision--a qualitative study in rural Mhondoro-Ngezi, Zimbabwe. Glob Public Health 2015, 10(5-6):708-720.

7. Sgaier SK, Reed JB, Thomas A, Njeuhmeli E. Achieving the HIV prevention impact of voluntary medical male circumcision: lessons and challenges for managing programs. PLoS Med. 2014;11(5):e1001641.

8. Prodger JL, Gray R, Kigozi G, Nalugoda F, Galiwango R, Hirbod T, et al. Foreskin T-cell subsets differ substantially from blood with respect to HIV co-receptor expression, inflammatory profile, and memory status. Mucosal Immunol. 2012;5(2):121-8.

9. Prodger JL, Gray RH, Shannon B, Shahabi K, Kong X, Grabowski K, et al. Chemokine levels in the penile coronal sulcus correlate with HIV-1 acquisition and are reduced by male circumcision in Rakai, Uganda. PLoS Pathog. 2016;12(11):e1006025.

10. Liu CM, Hungate BA, Tobian AA, Serwadda D, Ravel J, Lester R, Kigozi G, Aziz M, Galiwango RM, Nalugoda F et al: Male circumcision significantly reduces prevalence and load of genital anaerobic bacteria. mBio 2013, 4(2):e00076.

11. Liu CM, Prodger JL, Tobian AAR, Abraham AG, Kigozi G, Hungate BA, Aziz M, Nalugoda F, Sariya S, Serwadda D et al: Penile anaerobic dysbiosis as a risk factor for HIV infection. MBio 2017, 8(4).

12. Prodger JL, Kaul R. The biology of how circumcision reduces HIV susceptibility: broader implications for the prevention field. AIDS Res Ther. 2017;14(1):49.

13. Anderson D, Politch JA, Pudney J. HIV infection and immune defense of the penis. Am J Reprod Immunol. 2011;65(3):220-9.

14. Pudney J, Anderson DJ. Immunobiology of the human penile urethra. Am J Pathol. 1995;147(1):155-65.

15. Ganor Y, Zhou Z, Bodo J, Tudor D, Leibowitch J, Mathez D, et al. The adult penile urethra is a novel entry site for HIV-1 that preferentially targets resident urethral macrophages. Mucosal Immunol. 2013;6(4):776-86.

16. Ganor Y, Real F, Sennepin A, Dutertre CA, Prevedel L, Xu L, et al. HIV-1 reservoirs in urethral macrophages of patients under suppressive antiretroviral therapy. Nature Microbiol. 2019;4(4):633-44.

17. Pudney J, Anderson D. Innate and acquired immunity in the human penile urethra. J Reprod Immunol. 2011;88(2):219-27.

18. Mehta SD, Moses S, Agot K, Parker C, Ndinya-Achola JO, Maclean I, et al. Adult male circumcision does not reduce the risk of incident Neisseria gonorrhoeae, Chlamydia trachomatis, or Trichomonas vaginalis infection: results from a randomized, controlled trial in Kenya. J Infect Dis. 2009;200(3):370-8.

19. Tobian AA, Serwadda D, Quinn TC, Kigozi G, Gravitt PE, Laeyendecker O, et al. Male circumcision for the prevention of HSV-2 and HPV infections and syphilis. N Engl J Med. 2009;360(13):1298-309.

20. Weiss HA, Thomas SL, Munabi SK, Hayes RJ. Male circumcision and risk of syphilis, chancroid, and genital herpes: a systematic review and meta-analysis. Sexually Transmitted Infections. 2006;82(2):101-9 discussion 110.

21. Arnold KB, Burgener A, Birse K, Romas L, Dunphy LJ, Shahabi K, et al. Increased levels of inflammatory cytokines in the female reproductive tract are associated with altered expression of proteases, mucosal barrier proteins, and an influx of HIV-susceptible target cells. Mucosal Immunol. 2016;9(1):194-205.

22. Kigozi G, Wawer M, Ssettuba A, Kagaayi J, Nalugoda F, Watya S, et al. Foreskin surface area and HIV acquisition in Rakai, Uganda (size matters). AIDS. 2009;23(16):2209-13.

23. Kigozi G, Liu CM, Park D, Packman ZR, Gray RH, Kaul R, et al. Foreskin surface area is not associated with sub-preputial microbiome composition or penile cytokines. PLoS One. 2020;15(6):e0234256. 
24. Price LB, Liu CM, Johnson KE, Aziz M, Lau MK, Bowers J, et al. The effects of circumcision on the penis microbiome. PLoS One. 2010;5(1):e8422.

25. Baggiolini M, Clark-Lewis I. Interleukin-8, a chemotactic and inflammatory cytokine. FEBS Letters. 1992;307(1):97-101.

26. Harada A, Sekido N, Akahoshi T, Wada T, Mukaida N, Matsushima K. Essential involvement of interleukin-8 (IL-8) in acute inflammation. J Leukocyte Biol. 1994;56(5):559-64.

27. Liebenberg $L$, Masson L, Arnold KB, McKinnon LR, Werner L, Proctor E, et al. Genital-systemic chemokine gradients and the risk of HIV acquisition in women. J Acquired Immune Deficiency Syndromes. 2017;74(3):318-25.

28. Masson L, Passmore JA, Liebenberg LJ, Werner L, Baxter C, Arnold KB, et al. Genital inflammation and the risk of HIV acquisition in women. Clin Infect Dis. 2015;61 (2):260-9.

29. Shannon B, Gajer P, Yi TJ, Ma B, Humphrys MS, Thomas-Pavanel J, et al. Distinct effects of the cervicovaginal microbiota and herpes simplex type 2 infection on female genital tract immunology. J Infect Dis. 2017;215(9):1366-75.

30. Anton L, Sierra LJ, DeVine A, Barila G, Heiser L, Brown AG, et al. Common cervicovaginal microbial supernatants alter cervical epithelial function: mechanisms by which Lactobacillus crispatus contributes to cervical health. Front Microbiol. 2018;9:2181.

31. Furukawa F, Fujii K, Horiguchi Y, Matsuyoshi N, Fujita M, Toda K, et al. Roles of E- and P-cadherin in the human skin. Microscopy Research Technique. 1997;38(4):343-52.

32. Sierra LJ, Brown AG, Barila GO, Anton L, Barnum CE, Shetye SS, et al. Colonization of the cervicovaginal space with Gardnerella vaginalis leads to local inflammation and cervical remodeling in pregnant mice. PloS one. 2018;13(1):e0191524.

33. Garcia MA, Nelson WJ, Chavez N: Cell-cell junctions organize structural and signaling networks. Cold Spring Harbor Perspectives Biol 2018, 10(4).

34. Jiang YJ, Lu B, Crumrine D, Man MQ, Elias PM, Feingold KR. IL-1 alpha accelerates stratum corneum formation and improves permeability barrier homeostasis during murine fetal development. J Dermatol Sci. 2009;54(2):88-98.

35. Jung YJ, Jung M, Kim M, Hong SP, Choi EH. IL-1alpha stimulation restores epidermal permeability and antimicrobial barriers compromised by topical tacrolimus. J Investigative Dermatol. 2011;131(3):698-705.

36. Barland CO, Zettersten E, Brown BS, Ye J, Elias PM, Ghadially R. Imiquimodinduced interleukin-1 alpha stimulation improves barrier homeostasis in aged murine epidermis. J Investigative Dermatol. 2004;122(2):330-6.

37. Hauser C, Saurat JH, Schmitt A, Jaunin F, Dayer JM. Interleukin 1 is present in normal human epidermis. J Immunol. 1986;136(9):3317-23.

38. Gahring LC, Buckley A, Daynes RA. Presence of epidermal-derived thymocyte activating factor/interleukin 1 in normal human stratum corneum. J Clin Investigation. 1985;76(4):1585-91.

39. Fischetti L, Barry SM, Hope TJ, Shattock RJ. HIV-1 infection of human penile explant tissue and protection by candidate microbicides. AIDS. 2009;23(3):319-28.

40. Liu CM, Aziz M, Kachur S, Hsueh PR, Huang YT, Keim P, et al. BactQuant: an enhanced broad-coverage bacterial quantitative real-time PCR assay. BMC Microbiol. 2012;12:56.

41. Fadrosh DW, Ma B, Gajer P, Sengamalay N, Ott S, Brotman RM, et al. An improved dual-indexing approach for multiplexed 16S rRNA gene sequencing on the Illumina MiSeq platform. Microbiome. 2014;2(1):6.

42. Martin M: Cutadapt removes adapter sequences from high-throughput sequencing reads. 2011 2011, 17(1):3.

43. Bolger AM, Lohse M, Usadel B. Trimmomatic: a flexible trimmer for Illumina sequence data. Bioinformatics. 2014;30(15):2114-20.

44. Callahan BJ, McMurdie PJ, Rosen MJ, Han AW, Johnson AJ, Holmes SP. DADA2: high-resolution sample inference from Illumina amplicon data. Nat Methods. 2016;13(7):581-3.

45. Wang Q, Garrity GM, Tiedje JM, Cole JR. Naive Bayesian classifier for rapid assignment of rRNA sequences into the new bacterial taxonomy. Appl Environ Microbiol. 2007;73(16):5261-7.

\section{Publisher's Note}

Springer Nature remains neutral with regard to jurisdictional claims in published maps and institutional affiliations.

Ready to submit your research? Choose BMC and benefit from:

- fast, convenient online submission

- thorough peer review by experienced researchers in your field

- rapid publication on acceptance

- support for research data, including large and complex data types

- gold Open Access which fosters wider collaboration and increased citations

- maximum visibility for your research: over 100M website views per year

At BMC, research is always in progress.

Learn more biomedcentral.com/submissions 